\title{
Study on Control of Single Phase Grid-Connected Inverter Based on Fractional Order PI Controller
}

\author{
Ping Jiang ${ }^{1, a}$, Hai-jian Lv ${ }^{1, b^{*}}$ and Li-ying Wang ${ }^{1, c}$ \\ ${ }^{1}$ College of Electronic and Information Engineering, Hebei University, Baoding, China \\ a787111498@163.com, b1217190801@qq.com, '842292725@qq.com \\ *corresponding author
}

Keywords: single phase grid-connected inverter, fractional order $\mathrm{PI}^{\lambda}$ controller, Particle Swarm Optimization

Abstract: The inverter is a significant part in the single phase grid-connected inverter system. The design of control strategy for photovoltaic power generation system has a decisive role in the system. The fractional order $P I^{\lambda}$ controller is applied to the gridconnected inverter to improve the single phase photovoltaic grid-connected system performance, which is based on the integrated photovoltaic grid-connected inverter system in this paper. Three parameters of fractional order controller are optimized by Particle Swarm Optimization (PSO), and compared with the integer order PI controller. The results show that fractional order $P I^{\imath}$ controller can effectively reduce the harmonic components, and obtain better control effect than the integer order PI controller.

\section{Introduction}

The control strategy for the key link of inverters has become a hotspot [1-3]. Because the traditional PID control algorithm is easy to implement, the structure is simple and it is widely used in the control field [4]. But with the development of science and technology and computer technology as well as the improvement of modern industrial requirements, the traditional control algorithm has been unable to meet the control goals. A lot of new control algorithms appeared, for example, vector control of single phase grid-connected inverter has achieved the single phase power decoupling control strategy of voltage closed-loop, active current and reactive current closed-loop under grid voltage orientation in [5]. Sinusoidal signal integration method combines with instantaneous reactive power theory to detect active current and reactive current, which expands the function of the photovoltaic inverter in [6]. The multi-loop control strategy of current observation and repetitive control is studied to improve the steady-state accuracy and anti-jamming of the system in [7]. In this paper, the fractional order controller is applied in the control of PV gridconnected inverter, since the fractional order $P I^{\lambda}$ controller has a larger adjustment range $\lambda$ than the integer-order PI, which achieves the better results for the control of the inverter system. The parameters are optimized by PSO algorithm, significantly reducing the harmonic components. 


\section{Inner-Loop Current Control System of Single Phase Gird-Connected Inverter}

Inner-loop current control system uses voltage full-bridge inverter to complete the DC-AC invertion under full-bridge inverter circuit [8], the control structure is shown in Fig $1 . I_{\text {ref }}$ is the setting value of the current, $I_{\text {out }}$ is the output current of the inverter, after $I_{\text {ref }}$ and $I_{\text {out }}$ are operated by $\mathrm{PI}^{\lambda}$ controller, the result of the operation is added to the grid voltage feed forward to obtain the control duty cycle signal. The control signal is sent to the SPWM generator and SPWM signal drives the inverter switch to work, which makes the inverter output sine wave current and power grid have the same frequency and phase to achieve the control objectives [9].

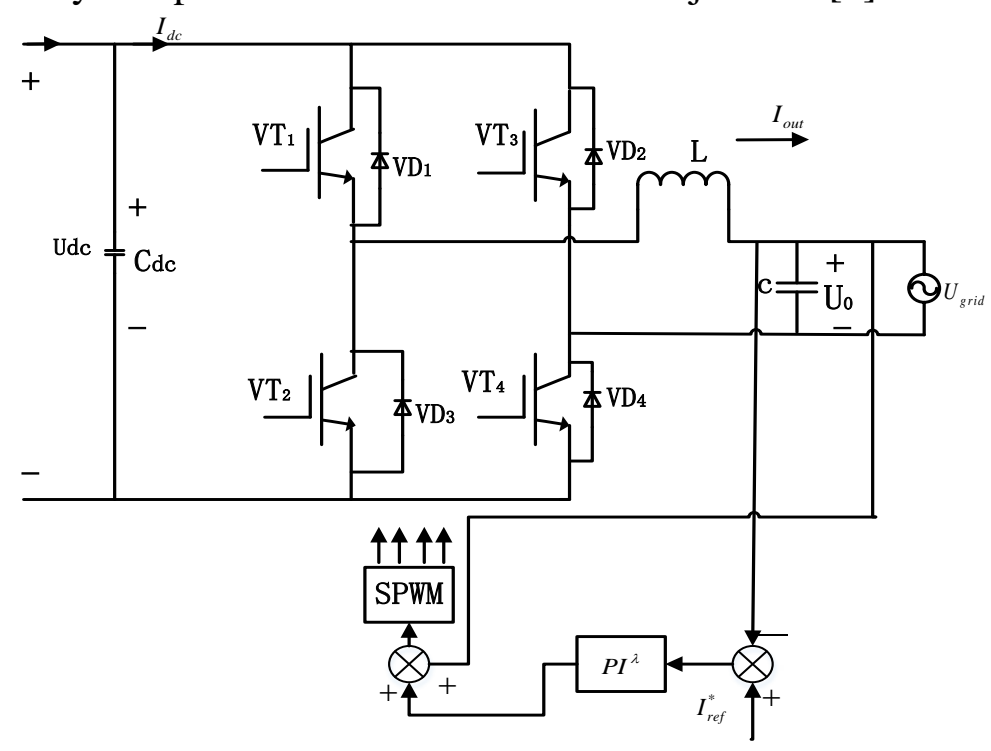

Figure.1 Control structure diagram of the inverter

\subsection{Mathematical Model of Current Inner-loop Control for Single Phase Gird-Connected Inverter}

Open loop transfer function of current inner is expressed as:

$$
G_{i o}(s)=G_{1}(s) G_{2}(s) G_{3}(s)=\left(K_{p}+\frac{K_{i}}{s^{\lambda}}\right)\left(\frac{K_{P W M}}{T_{P W M} s+1}\right)\left(\frac{1}{s L+R}\right)
$$

Where, $G_{1}(s)$ is the fractional order controller, $G_{2}(s)$ is the inverter link of SPWM control, $K_{P W M}=\frac{U_{d c}}{U_{c m}}$ is the gain of the inverter, and $U_{d c}$ is the DC bus voltage of DC side, $U_{c m}$ is the amplitude of carrier triangle wave, $T_{P W M}$ is the small inertia constant of the inverter, $G_{3}(s)$ is the transfer function of LC filter.

The control block diagram of the current inner loop is showed: 


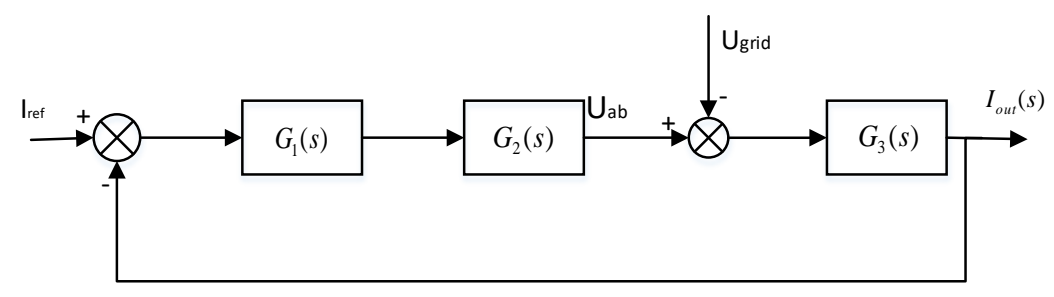

Figure.2 Block diagram current inner loop transfer function

In the current loop of the actual single phase grid-connected inverter control, the grid voltage contains harmonic components that can be seen as disturbance for the grid-connected current, then the inverter output current is decided by the given current and grid voltage at the same time [10].

In order to eliminate the interference of the grid voltage, feed forward compensation of the disturbance signal can be performed. As shown below:

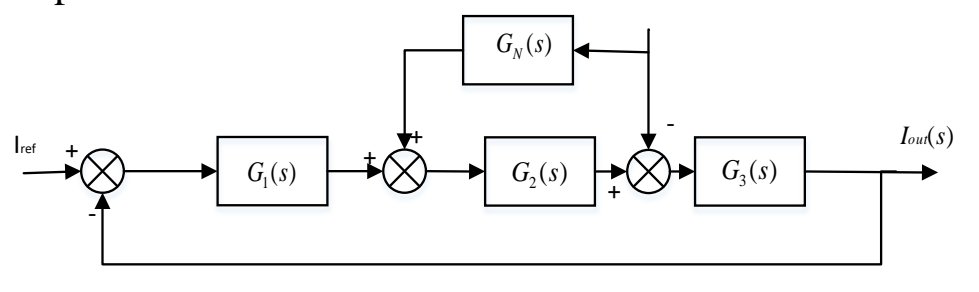

Figure.3 Block diagram of voltage feed forward compensation transfer function

Adding the voltage feed forward link, output response of grid voltage on Inverter Current is expressed as:

$$
I_{L G N}=\frac{G_{3}(s)\left(G_{2}(s) G_{N}(s)-1\right)}{1+G_{1}(s) G_{2}(s) G_{3}(s)} U_{g r i d}(s)
$$

If the disturbance is completely eliminated, then it is required that $G_{2}(s) G_{N}(s)-1=0$, namely, feed forward transfer function is:

$$
G_{N}(s)=\frac{1}{G_{2}(s)}
$$

\section{Fractional Order $P I^{\lambda}$ Controller}

Fractional order $\mathrm{PI}^{\lambda}$ controller is a generalized form of integer order PI controller, while integer order PI controller is a special form of fractional order $P I^{\wedge}$ controllers [11-12]. Related to the conventional PI controller, the fractional order $\mathrm{PI}^{\lambda}$ controller also introduces the $\lambda$ represented for integral order besides two parameters $\mathrm{Kp}$ and $\mathrm{Ki}$. In order to achieve the best control effect according to the different order of the controlled object to choose different values of $\lambda$. Since that the order can be continuously changed, so fractional order $P I^{\lambda}$ controllers have a greater flexibility than integer order controller. Block diagram of fractional order $P I^{\lambda}$ controller is: 


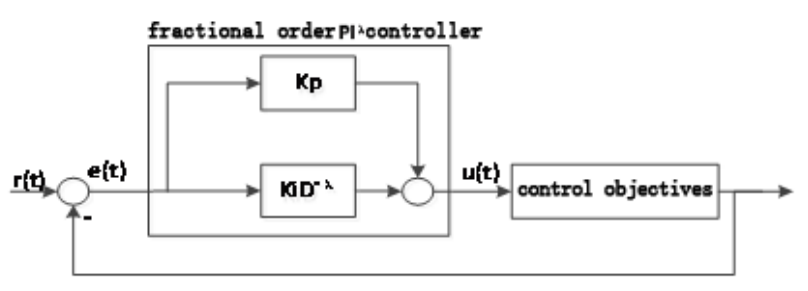

Figure.4 Block diagram of fractional order PID controller

Differential equation of fractional order $P I^{\lambda}$ controller is:

$$
u(t)=K_{p} e(t)+K_{i} D^{-\lambda} e(t)
$$

The transfer function of the controller obtained by Laplace Transform is:

$$
C(s)=U(s) / E(s)=K_{p}+K_{i} s^{-\lambda}
$$

Among the various successive filter approximations for fractional calculus, the Oustaloup filter has a quite remarkable performance [13], fractional order $P I^{\lambda}$ controller is realized by Oustaloup filter approaching to digital realization. Assume that the selected fit frequency segment is ( $\omega \mathrm{b}, \omega \mathrm{h})$, the transfer function model of continuous filter can be constructed:

$$
s^{\mathrm{r}}=H(s)=K \prod_{k=-N}^{N} \frac{s+\omega_{k}^{\prime}}{s+\omega_{k}}
$$

Where, $\omega_{k}^{\prime}=\omega_{b}\left(\frac{\omega_{h}}{\omega_{b}}\right)^{\frac{k+N+(1-r) / 2}{2 N+1}}, \omega_{k}^{\prime}=\omega_{b}\left(\frac{\omega_{h}}{\omega_{b}}\right)^{\frac{k+N+(1+r) / 2}{2 N+1}}, K=\omega_{h}^{r}$

Where, $r$ is the order of the fractional order, $2 \mathrm{~N}+1$ is the order of the filter, $(\omega b, \omega \mathrm{h})$ is the fit frequency segment. Generally, the fractional calculus operator can be well fitted in this region, but compared to the other region, the fitted result is much different from calculus operator.

\section{Optimize the Parameters Based on Particle Swarm Optimization}

The PSO algorithm is simple, easy to implement and has less parameter information, so that it has a better effect in continuous optimization problems [14]. Each individual of the population has a velocity (the change of the reaction position) in the PSO algorithm, the particle moves in the search space according to its velocity. Moreover, each individual has a memory cell to write down the optimal location it has ever reached to. The whole optimization process is the aggregation acceleration process that tends to the global optimal value, which the individual arrives at the optimal position and the others arrive at the optimal position and velocity [15].

PSO algorithm is used to optimize the parameters of fractional order $P I^{\lambda}$ controller, the steps are:

1) Initialize the position and velocity of all particles in the particle swarm.

2) Calculate the objective function of each initial particle.

3) Choose the best position of each particle and the best position of the whole population to compare with the optimal position adaptation value.

4) Update particle velocity and positions.

5) Judge whether it reaches the maximum number of iterations.

6) If it reaches the maximum number of iterations and it ends, while it does not reach the maximum number of iterations and it will be back.

\section{Simulation}

According to block diagram and its mathematical model of inner loop current control system of single phase gird-connected inverter, simulation circuit of single phase grid-connected inverter is 
built in Matlab / Simulink. Set the grid voltage to $220 \mathrm{~V}$ and set the peak voltage to 311V, direct current-bus capacitor C is $2200 \mu \mathrm{F}$, gird-connected filter inductor $\mathrm{L}$ is equal to $4 \mathrm{mH}$, equivalent internal resistance of the line $\mathrm{R}$ is $2 \Omega$, switching frequency of SPWM $f=20 \mathrm{kHZ}$, and $T_{P W M}=\frac{1}{20000}$,DC-side voltage is $380 \mathrm{~V}$, triangular carrier peak $U_{c m}=1 \mathrm{~V}$, $K_{P W M}=\frac{U_{d c}}{U_{c m}}=380$.According to the inner loop transfer function, and $G_{2}(s)=\frac{K_{P W M}}{T_{P W M} s+1}$, a small time constant $T_{P W M}$ is ignored, therefore $G_{2}(s)=\frac{1}{380}$.

PSO is used to optimize the parameters, and the parameters of the PSO algorithm are that inertia factor $\omega=0.6$, acceleration constant $c_{1}=c_{2}=2$, particle swarm size is set to 100 and the maximum number of iterations is 20. The ITAE error performance index was selected, namely $J=\int_{0}^{\infty} t|e(t)| d t$, the minimum fitness value is 0.01. The parameters of fractional order $P I^{\lambda}$ are optimized, which $K_{p}=27.0291, K_{i}=17.2264, \lambda=0.1$, the simulation results are shown below:

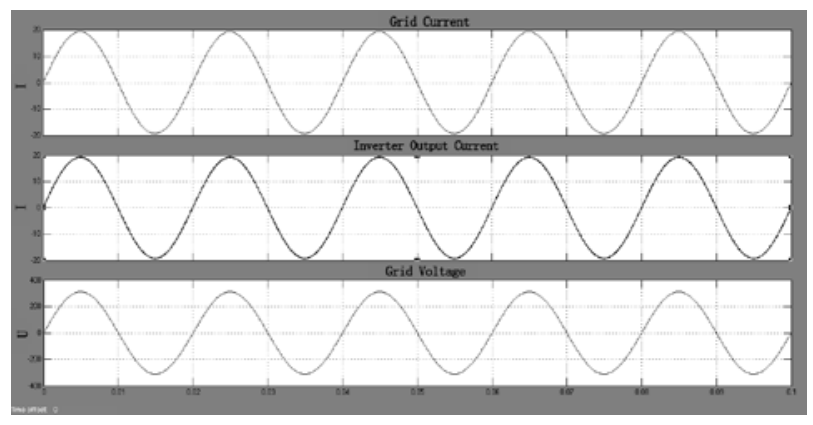

Figure.5 Current control under fractional order $P I^{\lambda}$ controller

Similarly, the optimal parameters of PI controller are optimized by PSO algorithm, which $K_{p}=29.7166, K_{i}=3.6366$, the simulation results are shown below:

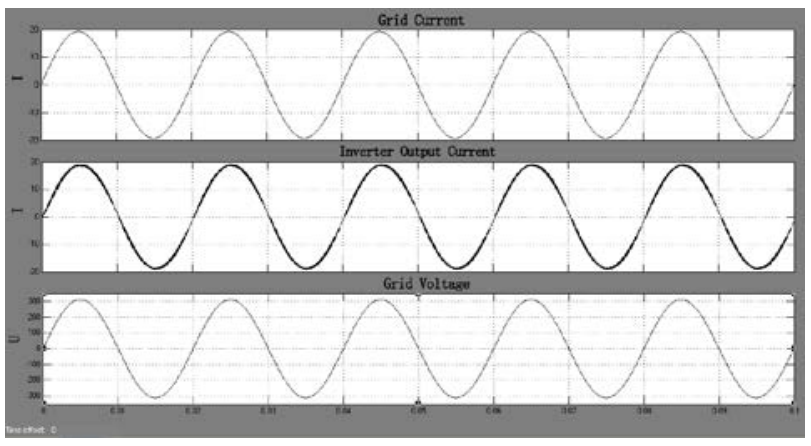

Figure.6 Current control under PI controller

The results show that the inverter can basically realize the output current to track the grid current under two kinds of control modes. Compared to the current waveform output of PI control, the output waveform is more smooth under $\mathrm{PI}^{\lambda}$ control than that, which reduce the harmonic components, and current tracking steady-state error is reduced in current control. So that the inverter output voltage has tracked better to the grid current. 
In order to compare the control effects of the two controllers more clearly, FFT analysis of the grid-connected output current is conducted under the two control modes. The results are shown below:

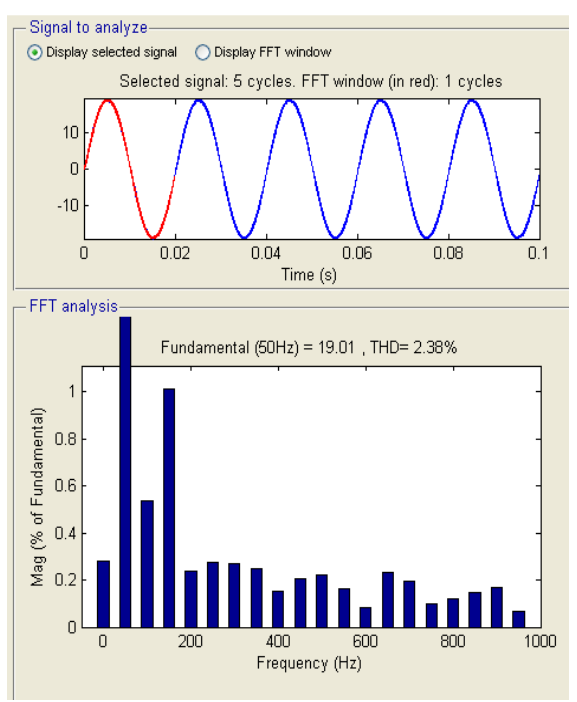

Figure.7 the FFT analysis of current output under PI control

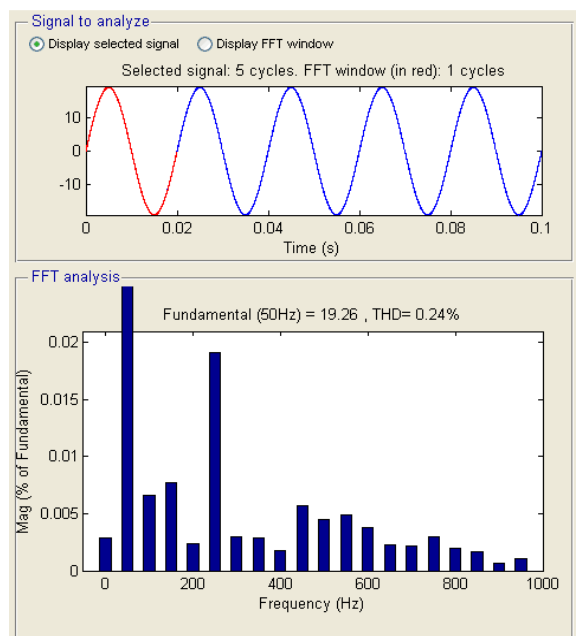

Figure.8 the FFT analysis of current output under control of fractional order $P I^{\lambda}$ controller

What the Figure.7 shows is the FFT analysis of current output under PI control, which $\mathrm{THD}=2.38 \%$ and the output current is $19.01 \mathrm{~A}$, and what the Figure.8 shows is the FFT analysis of current output under control of fractional order $P I^{\lambda}$ controller, which THD $=0.24 \%$ and the output current is $19.26 \mathrm{~A}$, it can meet the requirements for grid connection. Although adding the voltage feed forward link in the simulation and the grid voltage is regarded as the interference source of the system, it is obvious that the waveform still have more harmonic components under the PI control. However, fractional order $P I^{\lambda}$ controller can suppress the interference better than that, output stable sinusoidal current, which reduce the current tracking error.

\section{Conclusion}

This paper has analyzed the inner loop control system of single phase grid-connected inverter to propose the control target and the fractional order $\mathrm{PI}^{\lambda}$ control strategy. The comparison experiment 
has been conducted between the traditional PI controller and the fractional order $\mathrm{PI}^{\lambda}$ controller in the inner loop control of grid-connected inverter, what the result of simulation experiment shows is that the fractional order $P I^{\lambda}$ controller is able to make the single-phase grid-connected inverter reach the control target, reduce current tracking steady-state error and global harmonic components and make the system have a better steady-state characteristic. To sum up, this paper has achieved the goal that improves the stability and dynamic characteristics of the inverter output voltage.

\section{Acknowledgements}

This work was supported by the Post-graduate's Innovation Fund Project of Hebei University (X2016078).

\section{References}

[1] D. Cheng, Controllability of switched bilinear systems, IEEE Trans. on Automatic Control, 50(4): 511-515, 2005.

[2] YU Xiao-feng. Research on Single Phase Photovoltaic Gird-Connected Inverter and Its Control [D]. Liaoning University of Technology,2014.

[3] Wei DU, Qiong JIANG, Micah J. Erickson, et al. Voltage-Source Control of PV Inverter in a Certs Microgrid [J]. IEEE Transactions on Power Delivery, 2014,29(4):1726-1734.

[4] Peng Jun. Research and Design of Optimized Fractional Order PID Controller [D]. Shenyang: Northeastern University, 2009.

[5] FANG Gong-zhu, XIE Xin. Vector Control Strategy of Single-Phase Inverter. Telecom Power Technology, 2015,06:16-18.

[6] HU Yue, SHEN An-wen, TAN Hai-qing. Reactive Power Compensation Control Strategy of Single Phase Photovoltaic Grid- connected Inverter [J]. ELECTRIC DRIVE, 2015, 04:60-63.

[7] Lv Shi-xuan, ZHENG Li-jun, SONG Jian-cheng, DU Hai-long, MA Hai-xia. Study on Multi-Loop Control Strategy of Inverter Based on the Current Observation and Repetitive Control[J].ELECTRIC DRIVE,2015,11:3640.

[8] WANG Zhao-an, HUANG Jun. Power Electronics Technology [M].2000, Beijing: Machinery Industry Press.

[9] HUANG Jie-yin, LI Guang-sheng, XIE Yong-cheng, CHEN Long-long. Simulation of Low Power Single Phase Inverter with SPWM [J].Telecom Power Technology, 2015, 06:38-40.

[10] Xue Y, Chang L. Topologies of Single-Phase Inverters for Small Distributed Power Generators [J].IEEE Trans Power Electronics, 2004,19(5):1305-1314.

[11] SUN J. Impedance-Based Stability Criterion for Grid-Connected Inverters [J].IEEE Transactions on Power Electronics, 2011,26(11):3075-3078.

[12] LI Da-zi, LIU Zhan, JIN Qi-bing, CAO Liu-lin. Study on Optimization of Fractional-Order Controller PID Parameters[J]. Journal of System Simulation, 2007,19(19):4402-4406.

[13] QI Nai-ming, QIN Chang-mao, WANG Wei. Optimal Oustaloup Digital Realization of Fractional Order System Control and Decision, 2010,25(10): 1598-1600.

[14] YAO Shun-cai, PAN Hong-xia. Fractional Order PID Controller for Synchronous Machine Excitation Using Particle Swarm Optimization. Proceedings of the CSEE, 2010,30(21):91-97.

[15] HUANG You-rui. Intelligent Optimization Algorithm and Application[M].Beijing: National Defense Industry Press,2008. 